Rev. Elev. Méd. Vét. Pays trop., 1967, 20, I (121-124).

\title{
Note sur la salmonellose à Salmonella typhi-murium des oiseaux de cage au Sénégal
}

\author{
par M. P. DOUTRE, J. CHAMBRON ef F. SAGNA
}

\begin{abstract}
RÉSUMÉ
Salmonella typhi-murıum a été isolée des cadavres de nombreux Passeriformes appartenant soit à des amateurs, soit à un oiseleur exportateur imporiant. La formule antigénique de la souche en cause est de type classique. Les mesures de prophylaxie sanitaire appliquées d'une façon rigoureuse constituent le moyen le plus efficace de diminuer les pertes qu'occasionne cetle affection. Les agents thérapeutiques, quel que soit le degré d'efficacté, laissent tous demeurer des porteurs chroniques qui entretiennent la maladie.
\end{abstract}

Salmonella typhi-murium a été isolée chez de nombreux oiseaux appartenant aux espèces les plus diverses. Des états pathologiques liés à la présence de cette entérobactérie ont été maintes fois signalés chez la poule, le dindon, l'oie, le canard, la pintade ef le pigeon. Le diagnostic de paratyphose a été posé ou l'existence de porteurs de germes constatée chez la perdrix, le canard, les psittacidés, la grue, la movette, le moineau ef chez les olseaux de cage appartenant à des amateurs ou exposés dans des jardins zoologiques. L'affection a été également décrite chez différentes espèces sauvages vivant dans les conditions naturelles. Lorsque la maladie se déclare, les taux d'infection et de mortalité sont habituellement très élevés, les jeunes sujets payant le plus lourd tribut.

En 1965, nous avons eu l'occasion d'observer cette affection chez des oiseaux de volıère, propriété de quatre particuliers et d'un oiseleur spécialisé dans l'exporiation des espèces de I'Ouest-Africain vers l'Europe ef les Etats-Unis. Chaque année, du Sénégal, plusieurs centaines de milliers de petits passereaux sont acheminées par avion vers les marchés extérieurs et cette activité porte sur un chiffre d'affaires important. A titre d'exemple, en 1965, un seul commerçant s'est procuré par achat 615.171. parres d'olseaux ; sur ce nombre, 545.007 ont été vendues et 66.005 ont succombé avant l'expédition; le stock au 31 décembre s'établissait à 4.159 paires. Les exportations pour l'année 1965 se sont ainsi réparties : Pays-Bas : 146.831, Etats-Unis : 115.652, Allemagne de l'Ouest : 97.819, GrandeBretagne: 49.925 , Belgıque : 47.987, France : 45.595, Italie : 30.200 , divers (Suède, Liban, Malte, Uruguay) : 10.998:

Le développement de ceite activité peut être illustré par l'augmentation des envois vers les Etats-Unis effectués par cet exportateur : 1962 : 76.730 paires, $1963: 83.184,1964: 93.953$, $1965: 115.652$.

La maladie classique se présente soit sous une forme suraiguè, la mort survenant le plus souvent la nuif avant qu'aucun symptôme n'alt pu être observé, soit sous une forme aigue. Dans ce cas, l'oiseau se met « en boule », le plumage se gonfle et la diarrhée s'installe, une boulımie itrès nette se manifeste qui dure jusqu'aux derniers instants précédant l'agonie. Sous cette forme, la durée de l'évolution est d'environ 3 à 5 jours. La mortalıté des anımaux maintenus dans une même cage est importante et peut atteındre 100 p. 100.

Par hémoculture du sang du cœur, Salmonello typhi-murium a été isolée des cadavres des espèces 
suivantes, appartenant toutes au grand ordre des Passeriformes:

Serin du Mozambique (Sermus mozambicus). Chanteur d'Afrique (Serinus leucopygius).

Gendarme (Ploceus cucullatus).

Tisserin à lunettes (Ploceus brochypterus).

Ignicolore (Euplectes orix).

Monseigneur (Euplectes hordacea).

Worabée (Euplectes afra).

Veuve à collıer d'or (Steganura paradisea orientolis).

Beaumarquet (Pytilia melba).

Pytilie da ailes rouges (Pytiha phoenicoptera).

Senegali rouge (Lagonosticta senegala).

Bec de corail (Estrildo troglodytes).

Astrild à joves orangées (Estrilda melpoda).

Bengali cordon bleu (Uraeginthus angolensis).

Astrild d̀ ventre orange (Spoeraeginthus subflovus).

Queve de Vinaigre (Estrildo caerulescens).

Senegalı vineux (Lagonosticta rubricata).

Senegali à poitrıne barrée (Lagnosticta rufopicto).

Cou coupé (Amadina fosciata).

Combassou (Hypachera chalybeota).

Spermète à capuchon (Spermestes cucullatus).

Moineau ou Pinson doré (Auripasser luteus).

La formule antigénique de la souche de Salmonella typhi-murium isolée s'est révélée classique (I, II, V, XII : 1-2, I), la présence de l'antigène somatique $V$ permet d'éliminer la variété Copenhague si souvent rencontrée chez le pigeon. L'action sur les sucres n'offre rien de particulier : le glucose, le galactose, le lévulose, le mannitol et le sorbitol sont fermentés avec production de gaz, la dextrine, l'arabinose, le maltose, le raffinose et le xylose sans production de gaz, ne sont pas fermentés : le lactose, le saccharose, l'adonıtol, le dulcitol et l'inuline.

in vitro, la souche a manifesté une grande sensibilité à la framycétine, au chloramphénicol et à la colimycıne, une sensıbilité limite à la streptomycine, à la terramycine et à la polymyxine ; elle s'est montrée résistante à la tétracycline et à l'auréomycine.

La prophylaxie médicale par l'emploi de vaccin injectable est difficile à concevoir vu la taille des espèces atteintes, on peut néanmoins envisager l'utilisation d'un vaccin tué pour les oiseaux plus volumineux que les Passeriformes ; chez le pigeon, cette opération demeure possıble.

La protection la plus efficace d'une volière ou de cages non infectées réside dans l'application rigoureuse des règles de mise en quarantaine des oiseaux nouvellement acquis que I'on désire introduire.

Chez les oiseleurs, la maladıe semble exister d'une façon permanente et les sujets atteints, remis en lıberté ou qui s'échappent des cages, perpétuent l'affection, soit par contact direct avec les arrivants affaiblis par les conditions de transport, soit en souillant les aliments et l'eau de boisson. De plus, des poussières contaminées peuvent être ınhalées, ADLER et Coll. ont démontré que des poussins pouvaient être infectés par la voie intranasale (1). Dans les lieux d'entreposage des sacs de graines, la présence de rongeurs apporte une source de contamination supplémentare.

L'abandon cyclıque de certaines volières de stockage, leur nettoyage poussé suivi d'une désınfection rigoureuse, la destruction systématıque des oiseaux malades et de tous les agonisants qui encombrent les salles où se préparent les expéditions constituent des mesures qui doivent tendre d̀ lımiter le nombre des pertes. Salmonella typh-murium présente une résistance aux antıseptiques supérieure à celle des autres salmonelles, elle peut demeurer 119 jours dans l'eau des abreuvoirs (16) et 44 jours dans la paille des litières (1). Les temps maxima de persistance dans les sols varient de 120 à 150 jours (12), 110 à 159 jours (14), 251 ef 280 jours (8).

Les fumigations'd'aldéhyde formique, utilisées dans la désinfection des couveuses, apparaissent tout particulıèrement recommandées pour le traitement des volières d'entreposage étanches.

Les moyens therrapeutiques auxquels on peut faire appel sont variés. Le chlorhydrate de tétracycline doit être administré dans l'eau de boisson à la dilution de 0,01 p. 100, le chloramphénicol à celle de 0,04 p. 100 de chloramphénicol pur (6). Ces antibiotiques sont généralement fournis dilués au $1 / 10$ dans un excipient spécial, ce qui revient à dissoudre $1 \mathrm{~g}$ de tétracyclıne spécialité ef $4 \mathrm{~g}$ de chloramphénicol spécialité dans 1 litre d'eau de boisson.

Parmi les nitrofuranes, la furazolidone (6) insoluble peut être employée dans la graine entière ou mieux, dans de la graine broyée, à la concentration de 0,04 p. 100 de furazolidone 
base. La furaltadone $(2,3,12,15)$ soluble s'utilise dans l'eau de boisson ; du fait que cette substance accroît la soif, son absorption s'en trouve facilitée. La concentration recommandée varie de 0,04 p. 100 à 0,06 p. 100 . BURKHART et Coll. (5) préconisent jusqu'à $200 \mathrm{mg}$ de nitrofurane soluble par litre d'eau pour le traitement de canaris atteints de paratyphose.

Ces différentes substances présentent toutes, quel que soit leur degré d'efficacité, l'inconvénient majeur de laisser demeurer des porteurs chroniques (2). De plus, nombreuses sont les souches de Salmonella typhi-murium pour lesquelles la résistance aux dérivés sulfamidés, aux tétracyclines (2) (cas de la souche isolée à Dakar), au chloramphénicol (9) ef aux nitrofuranes $(2,4)$ a pu être mise en évidence in vitro et in vivo.
L'intérêt que présente la paratyphose à Salmonella typhi-murium des oiseaux de cage réside non seulement dans l'importance des pertes économiques que cette affection occasionne pour les oiseleurs, mais aussi dans le fait que les malades et les porteurs chroniques peuvent constituer une source de contamination pour l'homme et les autres espèces animales, volailles et mammifères domestiques.

\section{SUMMARY}

A Note on Salmonellosis of cage-birds caused by Salmonella typhi-murium in Senegal

S. typhi-murium was isolated from many Passeriformes belonging to bird fanciers or to a bird catcher who is doing export on a big scale.

The antigenic characteristics of the strain involved are of classical type.

Control measures, which have to be carried out strictly, are the most effective method in order to reduce the losses caused by the disease.

The therapeutic agents, whatever is their efflcacy, leave chronic carriers which are keeping the infection.

\section{RESUMEN}

Nota sobre la salmonelosis con Salmonello typhi-murium de los pájaros en jaula en Senegal

Se aisló Salmonella typhi-murium de numerosos passeriformes perteneciendo a aficionados, o a un pajarero, importante exporíador. Es de tipo clásico la fórmula antıgenica de la dicha cepa.

La profilaxıa sanitaria rigorosamente aplicada constiluye el medio más eficaz para disminuir la letalidad causada por la salmonelosis.

Ningún medicamento, cualquiera que sea su acción, impide la permanencia de portadores crónicos manteniendo esla enfermedad. 


\section{BIBLIOGRAPHIE}

1. ADLER (H. E.), NILSON (M. A.) et STADELMAN (W. J.). - A study of turkeys artificially infected with Solmonello typhimurium. Amer. J. vet. Res., 1953, 14 : 246.

2. BIERER (B. W.) et BARNETT (B. D.). Furaltadone water medication and the salmonellosis. Proc. XII World's Poultry Congr. Sydney, Sect. Papers, 1962, 283-285.

3. BIERER (B. W.), VALENTINE (H. D.) et VICKERS (C. L.), - Furaltadone water medication : its use in avian salmonellosis. Avion Dis., 1961, 5 : 214-218.

4. BIERER (B. W.) et VICKERS (C. L.). Nitrofuran medication for experimental Salmonella typhi-murium infection in poults. Avion Dis., 1960, 4 : 22-37.

5. BURKHART (D.M.), WOLFGANG (R.W.) et HARWOOD (P. D.). - Salmonellosis in parakeets and canaries treated with introfurans in the drinking water. Avian Dis., 1962, $6: 275-283$.

6. GRANVILLE (A.) et FIEVEZ (L.). - Clinical and bacteriological aspects of paratyphoid in birds. Ann. Méd. vét., 1962: 508-512.

7. LANNEK (N.), LINDGREN (N. O.) et NILSSON (T.). - Therapeutical experiments with a new nitrofuran compound (Tiafur) in Salmonellosis of chicks. Avian Dis., 1962, $6: 228-238$.

8. MAIR (N. S.) et ROSS (A. I.), - Sùrvival of Salmonella fyphi-murium in the soil. Mon. Bull. Minis. Hith. Lab. Serv., 1960, 19 : 39-41.
9. MANTEN (A.), KAMPELMACHER (E. H.) et GUINEE (P. A. M.). - Frequency of resistance to chloramphenicol and tetracycline among 13502 Salmonella strains isolated in 1961. Tijdschr. Diergeneesk, 1963, 88 : $411-417$.

10. NIELSEN (B. B.). - Salmonella typhi-murium infection in a flock of fowls as a source of infection for cattle. Proces. 9th Nordic Vet. Congr., Copenhogue, 1962, 1963, 1 : 339-342.

11. RAMSEY (C. H.) et EDWARDS (P. R.). Resistance of salmonellae isolated in 1959 and 1960 to tetracyclines and chloramphenicol. Appl. Microbiol., 1961, 9 : 389-391.

12. RICHEY (D. J.). - Water-soluble nitrofuran therapy in pullorum and fowl typhoid in chicks. Amer. J. vet. Res., 1962, 23 : 102-105.

13. SLAVKOV (I.). - Survival of Salmonella in soil. Proc. 2nd Symp. int. Ass. Vet. Food Hyg., Bosel, 1960, 279-283.

14. STEWART (D. J.). - The survival of Salmonella typhi-murium in soils under natural climatic conditions. Res. exp. Rec. Min. Agric. N. Ireland, 1962, $11: 53-56$.

15. TUCKER (J,F.). - The chemotherapy of avian salmonellosis with particular reference to furaltadone. Brit. vet. J., 1963, $119: 544-548$.

16. WATTS (P.S.) ef WALL (M.) - - The 1951 Salmonella typhi-murium epidemic in sheep in South Australia. Austr. vet. J., 1962, 28 : 165. 